\title{
LA ASOCIACIÓN ESPAÑOLA DE SEMIÓTICA (A.E.S.): \\ Crónica de una evolución científica
}

\section{José María Pozuelo Yvancos}

Presidente de A.E.S.

0. En mi condición de actual Presidente de la ASOCIACIÓN ESPAÑOLA DE SEMIÓTICA (A.E.S.) me corresponde hacer una breve historia de esta agrupación de científicos, que dé a conocer su contribución específica a los estudios semióticos internacionales, tarea que abordo con agrado, consciente de la enorme responsabilidad que con ello asumo, pero seguro de que toda disciplina y todo avance en cualquiera de ellas es hija de su propia historia y conviene tenerla presente. Para que tal historia no dependa en exceso (aunque algo será inevitable) de mi propia perspectiva, he adoptado un punto de vista lo más cercano posible a una historia científica: el análisis de los volúmenes de Actas de los diferentes Congresos, esto es, del conjunto de su producción intelectual como tal Asociación, dejando aparte, claro está, la de sus miembros individuales, que es cuantiosa y que ha sido seguida con detalle en bibliografías especializadas como las publicadas por José Romera Castillo de las que 
ofrezco una selección en la Bibliografía final, que se van actualizando con las diferentes addendas que este investigador viene publicando a sus bibliografías en números sucesivos de la revista Signa, órgano científico de la Asociación.

Mi contribución se limitará a glosar la que entiendo producción científica de la Asociación como tal, cuando ha actuado corporativamente con la organización y publicación de sus Actas, puesto que, lo adelanto ya, una de las singularidades más notables de la A.E.S. en el panorama científico internacional es que ha publicado hasta la fecha la totalidad de las Actas de sus VII Congresos, singularidad destacable no sólo por la rareza de que esto ocurra (en nuestro contexto científico de estudios semióticos será muy raro encontrar una fidelidad semejante en cualquier otra Asociación Internacional), sino también por el compromiso que supone con un estilo al hacer ciencia: toda reunión científica debe quedar, permanecer más allá de los breves días en que todo Congreso se desarrolla y contribuir a que tal Congreso, que publica lo que allí se debate, tenga por estilo propio también la responsabilidad de hacer frente a su propia historia, que permanece de ese modo ligada a un quehacer científico con conciencia de tal y no sólo a reuniones pasajeras o debates efímeros.

Antes de hacer tal análisis interno de las líneas científicas predominantes, permitáseme una breve historia externa de la Asociación.

\section{HISTORIA EXTERNA DE LA A.E.S.}

Entre los días 20 y 25 de junio de 1983 se celebró en el Consejo Superior de Investigaciones Científicas de Madrid el Congreso Internacional de Semiótica e Hispanismo, coordinado por Miguel Ángel Garrido Gallardo. Este Congreso tiene una doble importancia para la historia de la todavía entonces no nacida A.E.S: en primer lugar porque la iniciativa para el nacimiento de la Asociación se tomó en el seno de las sesiones de este Congreso; en segundo lugar porque fue un Congreso científico muy importante, por suponer la primera iniciativa de vinculación entre el mundo del Hispanismo y el de la Semiótica, dos mundos o dos comunidades científicas que tenían ya en las investigaciones de muchos de sus miembros puntos de contacto, pero que 
todavía no habían sido puestos en evidencia de manera explícita. La respuesta masiva a la convocatoria de ese Congreso, por parte de teóricos de la Semiótica e hispanistas venidos de muy diferentes países, indica que fue acertada la idea de tal vinculación.

Por iniciativa del profesor José Romera Castillo se convocó a una Asamblea constituyente de una Asociación Española de Semiótica, celebrada el día 23 de junio de 1983, en la cuarta jornada del Congreso. En esta Asamblea, que contó con la participación del prof. Cesare Segre, ponente del Congreso de Madrid y entonces Presidente de la International Association for Semiotic Studies (I.A.S.S-A.I.S), se eligió una Comisión organizadora del Primer Congreso de la A.E.S, que se habría de celebrar en Toledo un año más tarde. Dicha Comisión la formaron Jorge Lozano, Rafael Núñez Ramos, Cristina Peña-Marín, José Manuel Pérez Tornero, José Romera Castillo, Jenaro Talens y Jorge Urrutia.

Por consiguiente, aunque el Congreso de Semiótica e Hispanismo de Madrid (1983) no es propiamente una actividad de la A.E.S, tiene mucha importancia para su nacimiento y se encuentra vinculado a la vida de esta Asociación. No en vano en las que serán las primeras Actas de Congresos de la A.E.S (Investigaciones Semióticas I,1986: 225-232) Miguel Ángel Garrido Gallardo publicó una ponencia titulada «El primer Congreso Internacional sobre Semiótica e Hispanismo (1983)». Y no en vano, y quizá como reconocimiento a esta importancia la Primera Asamblea de la Asociación Española de Semiótica eligió a Garrido Gallardo como Presidente de la A.E.S. y al profesor Romera Castillo, que había tenido la iniciativa de la constitución de una Asociación Española de Semiótica, como Secretario General. Como en la historia interna que luego trazaré no me voy a referir a este Congreso de Madrid de 1983, remito al lector interesado a este artículo de Garrido donde se explican las líneas epistemológicas y las características científicas de aquel encuentro, así como al que aparece en este número de la revista.

El primer Congreso de la A.E.S se celebró en Toledo durante los días 7, 8 y 9 de Junio de 1984 y eligió la Primera Junta Directiva de la Asociación que formaron: Miguel Ángel Garrido Gallardo (Presidente), José Romera Castillo (Secretario), Alberto Álvarez Sanagustín (Vicepresidente), y Cristina Peña-Marín, José Manuel Pérez Tornero, Lorenzo Vilches y Alicia Yllera (Vocales). Se acordó celebrar en Oviedo, en el año 1986, el II Congreso Internacional, una periodicidad bianual que se ha respetado desde entonces, así como la 
publicación de las Actas de cada Congreso que son presentadas en el siguiente. También se impuso como práctica científicamente muy provechosa elegir un tema que focalizara las discusiones.

El II Congreso Internacional tuvo lugar en la Universidad de Oviedo entre el 13-15 de noviembre de 1986 y desarrolló el tema Lo cotidiano y lo teatral. Se eligió como Presidenta de la Asociación a la profesora Alicia Yllera, permanecieron en sus cargos de Vicepresidente y Secretario los profesores Álvarez Sanagustin y Romera Castillo y fueron vocales: J.M. Pérez Tornero, Lorenzo Vilches, Rafael Núñez y Jenaro Talens.

El III Congreso Internacional tuvo lugar en la Universidad Nacional de Educación a Distancia (U.N.E.D.) de Madrid entre los días 5 y 7 de diciembre de 1988, y giró en torno al tema Retórica y Lenguajes. Se reeligió a la profesora Alicia Yllera como Presidenta de la Asociación y a Popa-Lisseanu como Secretaria, y completaron la Junta Directiva los Vocales: Román Gubern (Vicepresidente), Jenaro Talens, Rafael Núñez, Antonio Sánchez Trigueros, José María Nadal, y se eligieron dos Vocales representantes de socios alumnos: Alfredo Martínez Expósito y J. Alonso Aldana.

El IV Congreso Internacional de la A.E.S. se celebró en la Universidad de Sevilla entre los días 3 y 5 de diciembre de 1990 . Se eligió al prof. José Romera Castillo como Presidente de la A.E.S., al prof. Alberto Álvarez Sanagustín como Vicepresidente y al prof. Mario García Page como Secretario. Completan la Junta Directiva como Vocales: Helena Usandizaga, A. Sánchez Trigueros, Jorge Urrutia, J. Javier Rubiera, Martínez Expósito y Jesús Corriente.

El V Congreso Internacional tuvo lugar en la Universidad de La Coruña, entre los días 3-5 de diciembre de 1992, sobre el tema de Semiótica y Modernidad. Resultaron reelegidos en sus cargos de Presidente y Secretario respectivamente, José Romera y Mario García Page y se incorporaron como Vocales los profesores J. M. Pozuelo Yvancos (Vicepresidente), José María Paz Gago, Sultana Wahnón, Helena Usandizaga, A. Álvarez Sanagustín, J. Urrutia y J. Corriente.

El VI Congreso Internacional se celebró en la Universidad de Murcia, entre los días 20-24 de noviembre de 1994, sobre el tema Mundos de ficción. Se eligió como Presidente a José María Pozuelo Yvancos, Vicepresidenta a Sultana Wahnón y Secretario a Francisco Vicente Gómez. Se incorporaron como Vocales a la Junta Directiva los 
profesores Wenceslao Castañares, Ana Recio y Mario García Page que pasó a Vocal.

El VII Congreso Internacional tuvo lugar en la Universidad de Zaragoza entre el 4-9 de noviembre de 1996 y giró en torno al tema de Mitos. Fueron reelegidos Presidente y Secretario, respectivamente, los profesores J. M. Pozuelo Yvancos y F. Vicente Gómez. Se eligió Vicepresidente al prof. Túa Blesa y se incorporaron como Vocales los profesores Fernando Cabo Aseguinolaza, Ana Recio Mir y W. Castañares.

El VIII Congreso Internacional tuvo lugar en la Universidad de Granada en el mes de diciembre de 1998, teniendo como tema Miradas y voces de fin de siglo.

La Asociación Española de Semiótica, como se deduce de esta breve historia interna, ha conseguido consolidar una periodicidad bianual de sus Congresos, la publicación hasta la fecha de todas sus Actas y la selección para sus temas de asuntos de amplio espectro semiótico que puedan suponer una respuesta científica a la vocación interdisciplinar que como Semiótica posee. Mantiene una relación científica continuada con las diferentes Asociaciones españolas e internacionales, de las que se da cuenta en otros artículos de este monográfico. Actualmente son socios de la Asociación unos trescientos profesores e investigadores de diecisiete países diferentes.

\section{HISTORIA INTERNA: PRINCIPALES DIRECCIONES CIENTÍFICAS DE LOS VII CONGRESOS INTERNACIONALES DE LA A.E.S. (1984-1996)}

Se podría trazar una breve historia de la evolución de la ciencia que conocemos como Semiótica a partir del estudio de los siete Congresos de A.E.S.. La primera evidencia de esta evolución es el progresivo ensanche de sus límites iniciales. Una prueba de tal ampliación de límites, que ha hecho a la Semiótica perder parte de su especialización inicial lo ofrecería el hecho de que el primer Congreso, celebrado en Toledo en junio de 1984 (Investigaciones Semióticas I, 1986), no tenía tema específico que especializase el estudio: las distintas ponencias de ese encuentro reunían todavía la ambición de un Congreso Semiótico de carácter general, donde las ponencias de carácter teórico, que ver- 
saron sobre Semiótica y filosofía o sobre Historia de la Semiótica, convivían con estudios sobre Pragmática, tanto general como literaria, sobre periodismo, etc. La enorme dispersión de intereses reflejaba al mismo tiempo que una amplitud de puntos de vista, la concepción de la Semiótica mucho más como herramienta metodológica, que proporcionaría las bases instrumentales para los análisis de diferentes discursos, que un objeto en sí mismo considerado, puesto que prevalecieron en ese Congreso los estudios de Semiótica aplicada, en que la Teoría Semiótica actuaba como fondo metalingüístico o como teoría base; todavía muy pocas ponencias se dedicaron al estudio o discusión de los presupuestos teóricos que revisasen u objetasen los fundamentos mismos de la teoría base, que se presuponían dados y comúnmente aceptados.

A esta primera evidencia deducible del análisis de las Investigaciones Semióticas I, sucede otra: la Semiótica pugnaba hace trece años entre dos tendencias o direcciones. La primera y muy potente, lo que será como veremos una constante en los estudios semióticos españoles, es la importancia que obtuvo desde los inicios la Semiótica Literaria, que en este mismo Congreso calificó el prof. Romera como salida del letargo para entrar en erupción (Investigaciones Semióticas I: $473 \mathrm{y} \mathrm{ss}$ ). Veinte de las cuarenta y seis intervenciones totales versaron sobre materias que tenían a la literatura como objeto. Una singularidad muy importante de la Asociación Española de Semiótica ha sido su contribución a la renovación de los planteamientos metodológicos de los estudios literarios en España. Con esto la semiótica española no ha hecho sino confirmar otra singularidad de la tradición filológica española: la poderosa compatibilidad que hubo en nuestro país entre estudios de carácter lingüístico y literario. Como ha sido puesto de relieve por E. Coseriu la escuela española de Filología tuvo siempre la especificidad en un contexto europeo de albergar una peculiar vinculación entre lengua y literatura. La tradición semiótica española no ha roto esta singularidad, dándose la circunstancia feliz a mi juicio de que buena parte de los jóvenes investigadores en textos, en teoría linguística, o en teoría literaria han presentado sus contribuciones renovadoras en los Congresos de la Asociación.

En esta dirección de semiótica literaria hay a su vez una doble vertiente a tener en cuenta: la simultánea serie de ponencias sobre aspectos metodológicos o metateóricos de carácter general: el concepto de intertextualidad, la etnosemiótica como mediación entre antropología y literatura, la enunciación narrativa, el diálogo en el relato, la prag- 
mática literaria, etc., con estudios de textos literarios concretos en que la narratología o la semiótica de la poesía o teatral eran discutidos como instrumento para análisis particulares de diferentes obras literarias del medievo, de la literatura áurea (siglos XVI y XVII) o de la contemporánea. Llamo la atención, puesto que estamos a la altura de 1984, sobre un hecho que tiene a mi juicio importancia y que definió el sesgo de las contribuciones a la teoría semiótica literaria en este Primer Congreso: la atención dedicada a la Pragmática literaria, que tuvo casi tanta importancia como la dedicada a la narratología. Lo segundo es más común en los Congresos internacionales, que han tenido, cuando se ha tratado de semiótica literaria, en el método narratológico su mayor desarrollo. Lo primero, el que los problemas de enunciación, de comunicación, de argumentación, de actos de habla, etc., tuvieran esa relevancia hace trece años era un índice de cambio de rumbo muy notable en esta parcela de los estudios semióticos; igual conclusión cabría extraer, en el terreno narratológico, de la crisis ya evidente de la llamada narratología de la historia (análisis funcionales y actanciales brillaron por su ausencia) en favor de la narratología del discurso, pero, insisto, siempre con menor relevancia que la obtenida por los procesos comunicativos y dialógicos.

Respecto a la semiótica no literaria, las líneas predominantes fueron de naturaleza semio-linguística, y de tipo formal, mucho más que los contextos ideológicos, todavía poco representados, aunque hubo ya dos ponencias dedicadas a ellos. De los discursos no literarios sometidos a análisis fueron los de naturaleza política y periodística los que recibieron atención.

El segundo Congreso de la Asociación, celebrado en la Universidad de Oviedo en noviembre de 1986 (Investigaciones Semióticas II, 1988, 2 vols.) supuso algunas variaciones notables con relación al primero. Quizá conscientes de la enorme dispersión metodológica del de Toledo, lo que unía a sus ventajas de mayor amplitud los inconvenientes de la enorme heterogeneidad de lenguajes y de objetos analizados, los organizadores propusieron dos temas monográficos, inaugurando así una tradición, mantenida hasta hoy, de que los Congresos de la A.E.S. tuvieran un tema (o dos) que centrase las intervenciones. La propuesta del doble tema de Lo cotidiano y lo teatral/Lo teatral y lo cotidiano, quizá respondía inicialmente a un intento por servir simultáneamente las que se habían mostrado dos direcciones potentes en el Congreso de Toledo: la literaria (entendiendo así, grosso modo, la semiótica teatral) y la de los discursos no literarios. En cualquier 
caso la inclusión de Lo cotidiano como tema del Congreso suponía también un intento, que también ha sido una constante de la A.E.S., por evitar una hegemonía de la semiótica literaria, que había sido estrella en el Congreso anterior, y que lo ha continuado siendo en los siguientes, si bien equilibrada consciente y programáticamente desde la propia organización proponiendo temas que vinculasen a los teóricos o estudiosos de los discursos no literarios.

La segunda novedad importante respecto al primer Congreso fue la invitación a Ponencias Generales sobre cada uno de los temas. Herman Parrett abrió con una ponencia sobre «Les temporalités du quotidien» (Investigaciones Semióticas II, 1988: 7-38) y Marco De Marinis inició el tema teatral con su ponencia: «Attraverso lo specchio. Per una ridefinizione dello spettacolo teatrale nei suo rapporti con il quotidiano» (Investigaciones Semióticas II, 1988: 9-40).

La orientación elegida por la ponencia de De Marinis merece comentario, puesto que supuso una de las singladuras destacables de este Congreso: frente a quienes habían concebido en él dos temas diversos, el de lo teatral por un lado y el de lo cotidiano por otro, De Marinis los vinculó. Pero curiosamente tal vinculación no la realizó sólo esta ponencia general, sino que fue muy frecuente encontrarla en las comunicaciones; de esta forma buena parte de ellas estudiaron tanto los asuntos cotidianos, como la intervención de la cotidianeidad en obras teatrales. Pero también se dio en numerosas comunicaciones el punto de vista contrario: el estudio de la intervención de la teatralidad en los comportamientos y en los signos cotidianos. Tal mixtura, tal contaminación de los dos temas, buscando sus puntos de convergencia, considero que resultó especialmente feliz y la destaco como una de las aportaciones más singulares de este Congreso, que puede ocupar, por tal circunstancia mixta, un lugar en la bibliografía de la semiótica teatral (comúnmente alejada de los aspectos de la cotidianeidad), como en la semiótica de los comportamientos (comúnmente alejada de la teatralidad como horizonte focalizado y como punto de vista).

El Congreso de Oviedo supuso asimismo un crecimiento cuantitativo muy notable, parejo con el que la A.E.S. iba experimentando en el número de sus socios, de forma que las Actas de este Congreso suponen ya dos gruesos volúmenes, con un total de novecientas cincuenta páginas, que recogen un total de sesenta y ocho participaciones.

El tercer Congreso de la Asociación se celebró en la Universidad Nacional de Educación a Distancia (U.N.E.D) de Madrid en diciembre 
de 1988 y tuvo como tema central el de Retórica y Lenguajes. (Investigaciones Semióticas III, 1990, J. Romera Castillo y Alicia Yllera, eds., 2 vols). Sus Actas recogen en dos gruesos volúmenes las tres sesiones plenarias, a cargo de Antonio García Berrio, M. Á. Garrido Gallardo y J. M. Klinkenberg, y las ciento diez comunicaciones leídas en ese Congreso. Respecto a la crónica de la evolución científica que vengo trazando, estas Actas marcan un hito muy importante: curiosamente, pese a ser el tema más especializado reunió a la mayor cantidad de participantes hasta ese momento en Congresos de la A.E.S., lo que significaba un continuo crecimiento de su actividad científica. Pero tratándose de un tema tan monográfico como el de la retórica estos dos gruesos volúmenes significan una monografía de referencia en las investigaciones retóricas en el mundo hispánico. Las dos circunstancias que acabo de glosar muestran asimismo la que considero la aportación más notable de ese Congreso: la concepción de la retórica como ciencia de la comunicación semiótica y ciencia de los discursos, mucho más allá de la retórica restringida de la elocución. Las tres conferencias plenarias son indicativas de las tres principales direcciones que organizarían luego el grueso de comunicaciones. García Berrio propuso una retórica general literaria, entendida como equivalente de una Poética General y su reflexión tendía a considerar la Retórica como pauta para la construcción de una ciencia del texto. Muchas de las comunicaciones también siguieron esta línea de reflexión metateórica sobre la relación entre Retórica y Semiótica Literaria.

La conferencia plenaria de M. Á. Garrido Gallardo, bajo el título de «Homo rhetoricus», se centró en las estructuras argumentativas y en la importancia que en la sociedades actuales tiene la retórica como pivote de las estructuras que sustituyen el concepto de verdad de la lógica modal, que significaría un sistema cerrado, por la verdad generada del discurso argumentativo, que es un sistema abierto e interpretable. En esta línea, otras comunicaciones versaron sobre los elementos persuasivos de los discursos periodístico y publicitario especialmente.

La conferencia plenaria de J. M. Klinkenberg, uno de los fundadores y animadores del famoso Grupo $M i$, de Lieja, que versó sobre la retórica de los mensajes visuales, supuso el planteamiento de unos fundamentos para la creación de una retórica general de la imagen. La importancia de este texto se señala por el hecho de que fue una de las primeras presentaciones públicas en foro científico de lo que luego fue un importante libro sobre retórica visual, que define una dirección poderosa de la Semiótica Internacional. 
El IV Congreso Internacional de la A.E.S. se celebró en la Universidad de Sevilla, en noviembre de 1990. Ha sido recogido en dos gruesos volúmenes (Investigaciones Semióticas IV, Madrid: Visor, 1992, 2 vols.) Una primera particularidad de este Congreso es que fue organizado conjuntamente con la Asociación Andaluza de Semiótica (A.A.S.), sociedad con un importante número de socios que celebra también bianualmente en universidades andaluzas diferentes Congresos. Buena parte de los socios de la A.E.S. también lo son de la A.A.S. y se encuentran por tanto doblemente comprometidos con el desarrollo de los estudios semióticos en España. En este Congreso hubo otro salto cuantitativo, pues el número de comunicaciones pasó a ser de ciento sesenta. Respecto a los dos anteriores supuso en la práctica una vuelta al modelo originario de tema abierto o libre, si bien con un intento de apoyar la dirección científica de la Semiótica de la Cultura al proponer como lema del Congreso Describir, inventar, transcribir el mundo, ordenado en cinco grandes apartados:

1. Semiótica general (treinta intervenciones).

2. Semiótica del espectáculo y medios de comunicación (veintinueve intervenciones).

3. Semiótica literaria (treinta y siete intervenciones).

4. Semiótica lingüística (nueve intervenciones).

5. Semiótica del Descubrimiento (seis intervenciones).

Aunque la Semiótica Literaria es en términos relativos la más numerosa, tenemos que anotar un giro muy considerable en el Congreso de Sevilla, pues a diferencia de los dos anteriores, el discurso literario no fue el predominante, antes bien tanto las intervenciones dedicadas a la Teoría Semiótica General como las dedicadas a la semiótica del espectáculo y medios de comunicación igualaron casi por separado a la literaria y si consideramos el conjunto de comunicaciones (ciento sesenta) las treinta y siete dedicadas al texto literario suponen un porcentaje muy inferior al de otros congresos de la Asociación. Particular interés tiene reseñar las dos series monográficas de semiótica lingüística (nueve intervenciones) y la sesión, puesto que se trataba de Sevilla y a dos años del Quinto Centenario del descubrimiento de América, dedi- 
cada monograficamente a Semiótica del Descubrimiento. Este último dato tendrá prolongación en el Congreso de La Coruña, dos años más tarde, y supuso ya el inicio de una andadura que la A.E.S. no ha abandonado desde entonces: la acogida en sus Congresos de los temas que tengan que ver con problemas intelectuales o preocupaciones históricas y sociales del entorno, lo que significa una concepción de la ciencia semiótica como intérprete también de los signos de cada momento y una ciencia semiótica alejada de cualquier dimensión autofocalizadora y de laboratorio, sustituible por una semiótica comprometida con los momentos históricos y culturales de la sociedad española.

Dos años más tarde, con una puntualidad que viene siendo uno de los signos de los Congresos de la A.E.S. y concretamente entre el 3 y cinco de diciembre de 1992, se celebró en la Universidad de La Coruña el V Congreso Internacional de la A.E.S, que respondió al título de Semiótica y Modernidad. (Investigaciones Semióticas V, 1994, J. M. Paz Gago, ed., 2 vols.). El que la apertura y cierre de este Congreso estuviera encargado inicial y respectivamente a I. Lotman (quien no pudo asistir, pero mandó su ponencia) y a U.Eco, dos figuras eminentes de la ciencia semiótica, da una idea de la resonancia que la creciente actividad de la A.E.S. ha venido teniendo. El número de intervenciones científicas fue de seis ponencias plenarias (J. P. Etienvre, D. Villanueva, C. Reis, I. Lotman, U. Eco, M. de Moragas) y setenta y nueve comunicaciones, agrupadas en los siguientes apartados:

1. Teoría semiótica y teoría de la literatura (dieciséis intervenciones).

2. La modernidad. Los signos del 92 (doce intervenciones).

3. Semiótica literaria: Modernidad y Postmodernidad (veinticuatro intervenciones).

4. Teoría y práctica de los métodos críticos (diez intervenciones).

5. Semiótica Visual: prensa, televisión, publicidad (doce intervenciones).

Tratándose de un Congreso celebrado en el año del Quinto Centenario del encuentro con América hubo una especial sensibilidad a la temática central de la Modernidad, pero también a la proyección latinoamericana, como quedó evidente no sólo en el tema de cuatro de las 
seis ponencias plenarias, sino en el hecho de que se organizara una mesa redonda en torno a la figura y obra del gran poeta Cesar Vallejo, de quien se cumplía el centenario. A esta mesa redonda, publicada, se añadieron otras tres dedicadas a «Los signos del 92» (coordinada por E. Montolió), «El otro centenario: las voces marginadas» (coordinada por S.Wahnón) y «Presente y futuro de la semiótica literaria» (coordinada por J. M. Pozuelo).

En la crónica científica que se está trazando, el Congreso de La Coruña supone dos incorporaciones muy notables: en primer lugar, una apertura de la propia concepción de la Semiótica, puesto que de una semiótica restringida a su propia teoría se ha pasado paulatinamente (ya vimos un atisbo de ello en los anteriores Congresos y acentuadamente en el de Sevilla) a una Semiótica entendida en el amplio sentido de «Signos culturales», esto es, de una ciencia puesta al servicio de la interpretación de la historia presente y pasada, con una vocación menos especulativa o metateórica y mucho más analítica. Paralelamente a ello, y como segundo rasgo incorporado netamente en el Congreso de la Coruña, se entiende la semiótica mucho menos como método particular heurístico y especializado en su propio metalenguaje para pasar a ser un lugar, un objeto de encuentro multidisciplinar. Esta evolución no nace en Coruña, se va viendo en los sucesivos Congresos de la A.E.S., pero emerge aquí con una evidencia muy contundente. En esta dirección creo que los primitivos modelos semiolinguíísticos están cediendo terreno en favor de la semiótica de la cultura. Que en concordancia con ello las Actas vengan dedicadas a la figura de I. Lotman, que no pudo venir al Congreso de Coruña por hallarse enfermo de la dolencia que acabaría con su vida poco después, es un gesto de completa coherencia.

Entre el 21 y 24 de noviembre de 1994 se celebró en la Universidad de Murcia el VI Congreso Internacional de la A.E.S., en torno al tema monográfico de Mundos de ficción (Investigaciones Semióticas VI, Universidad de Murcia, 1996, J. M. Pozuelo y F. Vicente, eds., 2 vols.). Como viene siendo habitual cuando se trata de proponer un tema monográfico los organizadores del Congreso quisimos que por la naturaleza del asunto, la ficcionalidad, pudiese ser abarcado desde diferentes puntos de vista y ciencias humanísticas. De hecho, pese a ser monográfico, el Congreso de Murcia supuso una participación muy crecida, puesto que hubo seis conferencias generales (a cargo de Lubomir Dolezel, Javier Echeverría, Félix Martínez Bonati, Thomas Pavel, Enrique Gil Calvo y Cesare Segre). Los temas de las conferencias abordaron la fic- 
cionalidad desde diferentes planos como el de los mundos posibles, el de la ciencia como constructora de ficciones, el de la literatura, el de la sociología. Se extendió la iniciativa del Congreso de Coruña de organización de mesas redondas sobre diferentes aspectos del tema de la ficcionalidad. En concreto se organizaron mesas redondas sobre «Ficción y Filosofía» (coordinada por F. Jarauta), «Ficción y Literatura» (coordinada por J. Domínguez Caparrós), «Ficción y discurso histórico» (coordinada por M. L. Meneghetti) y una mesa redonda de Homenaje a I. Lotman con la participación de Garrido Gallardo, J. Romera, A. Sánchez Trigueros y Cesare Segre. Si a ello unimos que las Comunicaciones presentadas superaron el número de doscientas, obtenemos como resultado unas Actas que en formato de letra pequeña ocupan dos gruesos volúmenes con un total de 1.563 páginas.

Temáticamente el Congreso sobre Mundos de ficción distribuyó sus sesiones en cinco grandes apartados: Ficción y Literatura, con el mayor número de participaciones, con lo que la ficción literaria y la semiótica que tiene como base el texto literario volvió a ser predominante en la línea de investigación de la A.E.S. Con todo, ello no fue óbice para una presencia interdisciplinar muy notable, sobre todo por la semiótica de las artes visuales (cine, televisión y publicidad) que fue el segundo tronco de importancia, junto a la Filosofía que tuvo una respresentación muy notable. Quizá como dato destacable es que se siguió acentuando el proceso de participación internacional iniciado en La Coruña, tanto por parte de los países latinoamericanos como por parte de investigadores europeos, ya que los participantes en el Congreso de Murcia provenían de catorce países distintos.

El VII Congreso Internacional, se celebró en Zaragoza entre el 4 y el 9 de noviembre de 1996. Estuvo dedicado al tema monográfico de Mitos y todavía tiene, en el momento de escribir este artículo (diciembre de 1997) las Actas en prensa, aunque el Coordinador general de dicho Congreso, Túa Blesa tiene ya muy avanzada su composición y fueron presentadas, como viene siendo habitual en los Congresos de la A.E.S., en el siguiente Congreso (el Octavo), celebrado en la Universidad de Granada, en diciembre de 1998 . No me es posible dar cuenta todavía del contenido de las Actas, ya que al escribir este trabajo aún no habían sido publicadas, aunque sí se puede dar una noticia del Congreso como tal. Desde el punto de vista de la crónica de la evolución científica que venimos haciendo el Congreso de Zaragoza supuso una profundización de la idea de combinar un tema monográfico, el Mito, con una participación interdisciplinar, de modo que éste fue estudiado como signo desde sus 
diferentes ángulos y protagonismos: filosofía, sociología, medios audiovisuales, literatura, historia, psicoanálisis, etc. Por darse la circunstancia de haber sido Zaragoza la ciudad donde se habían cumplido cien años de la primera filmación cinematográfica española, el Congreso dio un protagonismo especial a los mitos del cine.

Desde el punto de vista organizativo se potenciaron las Sesiones Plenarias de profesores invitados desde las diferentes ramas del saber, puesto que hubo catorce conferencias plenarias (a cargo de Ricardo Senabre, Carlos Castilla del Pino, Luis A. de Villena, J. Hillis Miller, Carlos García Gual, Germán Gullón, A. López, Elide Pittarello, J. Quetglas, A. Escohotado, Peer Aäge Brandt, Leonardo Romero Tobar, Leopoldo María Panero y Agustín Sánchez Vidal). Junto a las conferencias, que abordaron los diferentes aspectos del mito, se prosiguió con los paneles de ponencias plenarias, con profesores que intervenían en mesas redondas agrupadas por temas, lo que significó la participación en sesiones plenarias de unos cincuenta profesores provenientes de cuarenta universidades distintas de diez países diferentes. Ello dio a las sesiones plenarias sobre el Mito, celebradas en el magnífico Auditorio de Zaragoza, un gran relieve, puesto que el número de profesores inscritos que presentaron comunicaciones llegó a trescientos. Sin duda la Actas de este Congreso superarán las dos mil páginas, lo que ha supuesto uno de los Congresos Internacionales de Semiótica de mayor participación y dimensiones.

Desde el punto de vista de la evolución científica este Congreso de Zaragoza ha acentuado la dirección que la A.E.S. ha ido mostrando en los diferentes Congresos que en este artículo he resumido. Esto significa, a modo de síntesis, que la A.E.S. ha venido a entender la Semiótica cada vez más como campo de confluencia interdisciplinar de diferentes lenguajes que como un lenguaje científico uniforme o unidisciplinar. El concepto de signo se ha venido ensanchando, se ha concebido mucho más que como canal o como código, en el sentido global de comunicación de diferentes códigos y las ciencias que animan esos códigos y se han ido interpenetrando hasta llegar a unos Congresos que han perdido la especialización linguiístico-formal de la primitiva ciencia Semiótica y han devenido a la alternativa más imaginativa que el campo de la comuniación plantea: entender la semiótica como un lugar de encuentro de signos y códigos diferentes que generan una polifonía de mensajes. Definitivamnete creo que la llamada Semiótica de la Cultura va triunfando sobre las semióticas formales en el dominio investigador español. 
El VIII Congreso Internacional se celebró en la Universidad de Granada, en diciembre de 1998, coordinado por Antonio Sánchez Trigueros, sobre el tema Miradas y voces de fin de siglo, después de escrito este artículo.

\section{Referencias bibliográficas}

El repertorio bibliográfico más importante en referencia a la actividad de la A.E.S., son las diferentes Actas de los Congresos citados en el artículo y que responden a la siguientes fichas bibliográficas:

\section{ACTAS}

— Investigaciones Semióticas I. Actas del Primer Congreso de la Asociación Española de Semiótica. Madrid: C.S.I.C., 1986, 593 págs.

- Investigaciones Semióticas II. Actas del II Simposio Internacional de la Asociación Española de Semiótica. Lo teatral y lo cotidiano/Lo cotidiano y lo teatral. Oviedo: Universidad de Oviedo, Servicio de Publicaciones, 1988, 2 vols.

- Investigaciones Semióticas III. Retórica y Lenguajes. Actas del III Simposio Internacional de la Asociación Española de Semiótica. J. Romera Castillo y A. Yllera (eds.). Madrid: U.N.E.D., 1990, 2 vols.

- Investigaciones Semióticas IV. Describir, inventar, transcribir el mundo. Actas del IV Simposio Internacional de la Asociación Española de Semiótica. Madrid: Visor Libros, 1992, 2 vols., 1.055 págs.

- Investigaciones Semióticas V. Semiótica y Modernidad. Actas del VI Congreso Internacional de la Asociación Española de Semiótica. J. M. Paz Gago (ed.). Universidade da Coruña, 1994, 2 vols.

- Investigaciones Semióticas VI. J. M. Pozuelo Yvancos y F. Vicente Gómez (eds): Mundos de ficción. Actas de VI Congreso Internacional de la Asociación Española de Semiótica. Murcia: Universidad de Murcia, Servicio de Publicaciones, 1996, 2 vols., 1.563 págs.

\section{Panoramas}

José Romera CASTLLo ha venido publicando diferentes estados de la cuestión sobre Semiótica literaria y teatral en España. Entre ellos pueden consultarse:

- (1985). «Panorama de la critica semiótica de la literatura en España (19791983)». En Teoría Semiótica. Lenguajes y Textos hispánicos, M. Á. Garrido Gallardo (ed.), 433-456. Madrid: C.S.I.C. 
- (1986). «Semiótica literaria en España (Del letargo a la erupción)». En Investigaciones Semióticas I, 473-488.

- (1988 a). «Semiótica teatral en España». En Investigaciones Semióticas II, 353-388.

- (1988 b). Semiótica literaria y teatral en España. Kassel: Reichenberger.

- (1989). «Semiótica literaria y teatral en España: Addenda bibliográfica I». Letras de Deusto 44, 269-286.

- (1990). «Semiotica literaria y teatral en España: addenda bibliográfica (1 y 2)» y «Semiótica teatral en español: ampliación bibliográfica». En Investigaciones Semióticas III, 537-571.

- (1991). «Semiótica literaria y teatral en España: addenda bibliográfica III». Discurso 6, 107-134.

- (1992). «Semiótica literaria y teatral en España: addenda bibliográfica IV». En Investigaciones Semióticas IV, 1.043-1.055.

- (1993). «Semiótica literaria y teatral en España: addenda bibliográfica V». En Signa 2, 167-184.

- (1995). «Panorama del análisis semiótico del cuento en España». En P. Fröhlicher y G. Güntert (eds.), Teoría e interpretación del cuento, 103124. Berna: Peter Lang ( $2 .^{\circ}$ ed., 1996).

- (1998). Literatura, teatro y semiótica: Método, prácticas y bibliografia. Madrid: UNED. 